\title{
2 Grundlagen des Managements der sozialen Verantwortung von Unternehmen
}

\author{
Silke Bustamante
}

\subsection{Das Konzept der sozialen Verantwortung von Unternehmen}

\subsubsection{Der Begriff der Verantwortung in der Unternehmensethik}

Verantwortung wird verstanden als „Eintreten(müssen) eines Subjekts für ein Objekt“ (Fetzer 2004, S. 88). Sie gilt als fundamentale Forderung der Unternehmensethik (Göbel 2006, S. 99), welche sich mit der Frage nach den richtigen Normen und Wertvorstellungen, denen Unternehmen genügen sollten, beschäftigt ${ }^{5}$.

Mit dem Subjekt wird auf die Frage geantwortet, wer Verantwortung trägt. Im Bereich der Unternehmensverantwortung sind dies im Wesentlichen die Geschäftsführung, sonstige Führungskräfte und die Mitarbeiter, die für ein Unternehmen handeln, aber auch die Institution „Unternehmen“ selbst (Enderle 1988, S. 56).

5 In diesem Zusammenhang gibt es unterschiedliche Auffassungen über die „richtigen“ Normen und Wertvorstellungen, welche sich aus allgemeinen ethischen Prinzipien, dem Prinzip der Vernunft oder dialogisch aus den Ansprüchen der Verantwortungsadressaten ableiten lassen können (vgl. hierzu zum Beispiel Homann/Gerecke (1999), Ulrich (2001) und Steinmann/Löhr (1992)).
Bei dem Verantwortungsobjekt geht es einerseits um Aufgaben, Handlungen, Unterlassungen oder Entscheidungen und deren Folgen, aber auch um die Adressaten dieser Handlungen (man selbst oder andere, welche durch das Tun oder Lassen betroffen sind). Wesentlich für den Begriff der Verantwortung ist das Vorliegen einer Verantwortungsrelation (das heißt eine Einflussmöglichkeit des Subjektes auf das Objekt) sowie einer Instanz, vor der man sich zu verantworten hat (z.B. Gerichte, Öffentlichkeit, Gott, Vernunft, Gewissen) (Göbel 2006, S. 102).

Verantwortliches Handeln setzt Handlungsspielräume voraus, innerhalb der die Handelnden, unter Berücksichtigung ethischer Überlegungen und der Interessen der durch die Handlungen betroffenen Personen, Entscheidungen fällen (z.B. Küpper; Picot 1999, S. 132). Diese Handlungsspielräume sind nur gegeben, wenn das Unternehmen nicht von externen Instanzen (insbesondere von Gerichten), zur Verantwortung gezogen wird“, sondern freiwillig und eigenverantwortlich für die Folgen seines Handelns eintritt (z.B. Fetzer 2004, S. 200).

Nicht nur Gerichte können Unternehmen zur Verantwortung ziehen, sondern auch die Öffentlichkeit, welche Anforderungen an das Verhalten und gesellschaftliche Engagement 
von Unternehmen stellt und Unternehmen kritisiert, kontrolliert und sanktioniert (Göbel 2006, S. 106). Fraglich ist in diesem Zusammenhang, ob, wann bzw. in welchem Grad dem Unternehmen dennoch Handlungsspielräume bleiben, so dass man von freiwilliger Verantwortungsübernahme sprechen kann. Wenn der Öffentlichkeit bzw. den Konsumenten ökonomische oder andere Sanktionen zur Verfügung stehen, durch welche sie Unternehmen zu erwartungskonformen Verhalten zwingen können, könnte man dies in Abrede stellen. Allerdings spielt die soziale Kontrolle eine entscheidende Rolle für die Durchsetzung ethischer Orientierung und es unterscheidet sich der ethische Koordinationsmechanismus über soziale Sanktionsmechanismen von dem Zwang rechtlicher Normen (Schreyögg; Koch 2007, S. 60). Grundsätzlich wird daher im Folgenden davon ausgegangen, dass Erwartungen der Öffentlichkeit und einzelner Personen oder Personengruppen normativ nicht so verpflichtend sind, dass dadurch der Charakter der Freiwilligkeit in Abrede gestellt werden muss.

Der Verantwortungsbegriff berührt unterschiedliche Typen ethischer Argumentation. Insbesondere stellt sich die Frage, ob sich Verantwortung im Wesentlichen auf die Folgen des Handelns bezieht, oder ob auch die Absicht bzw. das Handeln nach sittlich anerkannten Maßstäben für die Bewertung menschlichen bzw. unternehmerischen Handelns relevant sind ${ }^{6}$.

Berücksichtigt man alle drei Aspekte, so bedeutet Unternehmensverantwortung, ,dass sich Subjekte (Unternehmensmitglieder sowie die Unternehmung selbst) für ihr Tun und Lassen und die Folgen daraus [freiwillig] verantwortlich erklären und sich gemäß dieser Verantwortung auf der Grundlage sittlicher Verbindlichkeiten richtig verhalten wollen" (Göbel 2006, S. 104). Eine derart verstandene Definition von Verantwortung beinhaltet einerseits das freiwillige Eintreten für die Folgen von Handlungen, andererseits aber auch das gewollte Handeln nach verbindlichen sittlichen Grundsätzen.

\subsubsection{Soziale Verantwortung - der Bezug auf die Stakeholder}

Der Begriff sozial (von lat. Socius = gemeinsam, verbunden, verbündet) bezeichnet die wechselseitigen Bezüge (Prozesse, Handeln bzw. daraus erwachsenden Institutionen), die in den Cesamtzusammenhang der Gesellschaft eingelagert sind (Hartfiel; Hillman 1972, S. 695). In der Umgangssprache bedeutet sozial den Bezug einer Person (bzw. eines Unternehmens) auf eine oder mehrere andere Personen.

Im Zusammenhang mit der Unternehmensverantwortung kann sich der Begriff „sozial“ auf soziale Belange bzw. Themen beziehen, für welche sich ein Unternehmen verantwortlich erklärt, oder aber auf die Adressaten als Personen und Personenkreise, mit welchen das Unternehmen in einer sozialen bzw. wechselseitigen Beziehung steht. Dies sind bei Unternehmen zum Beispiel Mitarbeiter, Kunden, Lieferanten, Gläubiger, der Staat und gesellschaftliche Cruppierungen (Umweltschutzverbände, Verbraucherschutzverbände u.ä.) bzw. bei Krankenhäusern die Krankenhausbelegschaft, Patienten, niedergelassene Ärzte und Krankenkassen. In der Regel besteht zwischen Unternehmen und der entsprechenden Person bzw. Personengruppe ein einseitiger oder gegenseitiger Anspruch, ein Verlustrisiko bzw. zumindest ein Interesse an dem Bestand und der Weiterentwicklung des Unternehmens, sodass sich für letztere die Bezeichnung "Stakeholder“ eingebürgert hat ${ }^{7}$. Da die Verantwortung für soziale Belange in der Regel durch die Stakeholder eingefordert wird, soll im Folgenden der Bezug der Unternehmensverantwortung auf die Stakeholder des Unternehmens im Vordergrund stehen.

Soziale Verantwortung von Unternehmen bedeutet dann, dass Unternehmen bzw. ihre Mitglieder sich (freiwillig) verantwortlich erklären für ihr Handeln bzw. dessen Auswirkungen auf das soziale Gefüge, welches durch die Stakeholder ausgedrückt wird und sich gemäß dieser Verantwortung auf der Crundlage sittlicher Verbindlichkeiten richtig verhalten wollen. Da Unternehmen sich in der Regel mit einer Vielzahl von Personen und Personengruppen mit unterschiedlichen Interessen und Ansprüchen konfrontiert sehen, für welche sie in unterschiedlichem Ausmaß Verant-

7 Von "to have a stake in something“. Vgl. zum Konzept des Stakeholder-Ansatzes Freeman, 1984 sowie Kapitel 2.2.2.
6 Es geht hier im Wesentlichen um Argumentationsmuster der Gesinnungs-, Pflichten- und Folgenethik. 
wortung tragen, sind bei Interessen- und Ressourcenkonflikten Entscheidungen zu treffen, welche Ansprüche und Interessen mit welcher Priorität behandelt werden. Laut dem Grünbuch der EU handelt ein Unternehmen dann verantwortlich, „wenn es anstrebt, ein für alle Beteiligten akzeptables Gleichgewicht zwischen den Erfordernissen und Bedürfnissen der verschiedenen Stakeholder herzustellen" (Europäische Kommission 2002, S. 4). Was dabei akzeptabel ist und wie ein solches Gleichgewicht hergestellt wird, kann nur auf Basis einer eingehenden Stakeholderanalyse und mit Rückgriff auf ethische Prinzipien bzw. Maßstäbe ${ }^{8}$ entschieden werden (s. Kap. 3.3.3).

\subsubsection{Soziale Verantwortung von Unternehmen als Managementkonzept}

Soziale Verantwortung von Unternehmen (auch bezeichnet als Corporate [Social] Responsibility) wird in der politischen und betriebswirtschaftlichen Diskussion inzwischen als Konzept der Unternehmensführung begriffen, mittels dessen verantwortliches Verhalten im Unternehmen verankert werden kann.

In dem Grünbuch der Europäischen Kommission wird soziale Verantwortung von Unternehmen definiert als „ein Konzept, dass den Unternehmen als Crundlage dient, auf freiwilliger Basis soziale Belange und Umweltbelange in ihre Unternehmenstätigkeit und in die Wechselbeziehungen mit den Stakeholdern zu integrieren “ (Europäische Kommission 2001, S. 8), wobei ein „für alle Beteiligten akzeptables Gleichgewicht zwischen den Erfordernissen und Bedürfnissen der verschiedenen Stakeholder herzustellen [ist] “ (Europäische Kommission 2002, S. 4). Die gesamthafte Steuerung ökologischer, sozialer und ökonomischer Aspekte dient dabei erstens der Erreichung einer nachhaltigen Unternehmens- und Geschäftsentwicklung und zweitens der Sicherstellung eines positiven Beitrag des Unternehmen zur nachhaltigen Entwicklung der gesamten Gesellschaft (Europäische Kommission 2002, S. 9). Wesentlicher Bestandteil des Konzeptes sind folglich die Freiwilligkeit (das heißt über gesetzliche Verpflichtung hinausgehende Aktivitäten), der

8 Unter ethischen Maßstäben werden dabei Maßstäbe bzw. Kriterien verstanden, welche auf allgemeingültigen Werten und Normen beruhen bzw. allgemein anerkannt sind.
Bezug auf das aus der Nachhaltigkeitsdiskussion stammende drei Säulen Prinzip sowie die Orientierung an den Erwartungen und Werten der Stakeholder eines Unternehmens.

Im folgenden wird soziale Verantwortung daher verstanden werden als integrierendes Unternehmenskonzept, welches unter Berücksichtigung unternehmensspezifischer Ziele, Werte und Kompetenzen sowie der Interessen relevanter Stakeholder verantwortungsvolles Handeln ganzheitlich im Unternehmen verankert und alle über gesetzliche Bestimmungen hinausgehende sozialen, ökologischen und ökonomischen Beiträge eines Unternehmens zur freiwilligen Übernahme gesellschaftlicher Verantwortung beinhaltet (Meffert; Münstermann 2005, S. 22).

\subsection{Die Akteure der sozialen Verantwortung von Unternehmen}

\subsubsection{Verantwortungsträger}

Wie in Kapitel 2.1.1 angesprochen, können sowohl das Unternehmen selbst als auch die für das Unternehmen in ihrer Rolle als Führungskräfte oder Mitarbeiter handelnden Individuen als Verantwortungssubjekte gesehen werden. Mitverantwortung tragen außerdem all jene Wirtschaftsakteure, welche indirekt das Unternehmenshandeln beeinflussen, z.B. Konsumenten, Eigentümer bzw. Investoren (sofern sie nicht selbst in der Geschäftsführung des Unternehmens involviert sind) und Politiker (Göbel 2006, S. 100). Nicht zuletzt prägt die vorhandene Rahmenordnung, innerhalb derer sich Unternehmen bewegen, die Entscheidungen der in ihr handelnden Unternehmen und schreibt ihnen bestimmte Rollen zu (ebenda, S. 96).

Unternehmen sowie die in ihnen handelnden Individuen tragen Aufgabenverantwortung (das heißt die Zuständigkeit für bestimmte Funktionen, Ämter und Rollen) sowie Handlungsverantwortung (das heißt die Zuständigkeit für die Folgen und Nebenfolgen des eigenen Tun und Lassens) (Höffe 2008, S. 326f.) und sind für die Wahrnehmung und Berücksichtigung von Stakeholderanliegen verantwortlich.

Die Verantwortungsfähigkeit und -notwendigkeit von Unternehmen wird dabei wie folgt begründet:

- Einerseits kann das Unternehmen als besondere Art von Subjekt mit stabiler Identität ge- 
sehen werden, welches intentional handelt und dadurch Wirkungen auf Dritte hervorruft und dessen innere Struktur das Entscheiden und Handeln der Individuen in der Unternehmung maßgeblich beeinflusst (z.B. Enderle 1992, French 1979).

- Andererseits kann man argumentieren, dass Unternehmen einen institutionellen Rahmen für die in ihr handelnden Individuen schaffen und auf diese Weise Verantwortung tragen (i.d.S. Göbel 2006, S. 89).

- Schließlich sind Unternehmen als Verantwortungsträger notwendig, da Folgen einer Handlung nicht immer natürlichen Personen zugerechnet werden können und potenziell Geschädigte in diesem Fall nur von Unternehmen Ausgleich erhalten können.

Die Verantwortungsübernahme der für das Unternehmen handelnden Individuen ergibt sich aus der Tatsache, dass diese nicht gleichsam willenlos innerhalb einer gegebene Struktur agieren, sondern die innere Struktur des Unternehmens mit gestalten, ihren Regeln Folge leisten, sie hinterfragen und Lücken und Mehrdeutigkeiten in den Rahmenbedingungen überbrücken. Darüber hinaus können Individuen auch durch Entscheidungen und Handlungen, welche nicht in der Struktur berücksichtigt sind, als Vorbildfunktion fungieren.

Die Verantwortung des Unternehmens und der in ihm handelnden Individuen stehen also in einem wechselseitigen Zusammenhang. Das Unternehmen und dessen innerbetriebliche Rahmenordnung (Organisationsstruktur, Prozesse, Anreize, Unternehmenskultur) ist also Medium des Handelns und beeinflusst das Handeln der Individuen, gleichzeitig ist dessen Struktur aber auch Resultat des Handelns der Individuen, die diese reproduzieren, ergänzen oder ändern?

Konsumenten, Eigentümer, Investoren und Politiker sind in der Regel nicht nur Mitträger der Verantwortung, sondern gleichzeitig auch ihre Adressaten (s. Kap. 2.2.2); auch können sie ihren Einfluss nur indirekt geltend machen. Im Folgenden wird daher das Unternehmen und die im Unternehmen handelnden und entscheidenden

9 Konzept der „Dualität von Struktur“ nach Giddens (1988). Vgl. dazu auch Albach, 2005, der Unternehmensethik als Ethik des Individuums auf der einen Seite und Ordnungsethik auf der anderen Seite begreift.
Personen als Träger und sonstige Wirtschaftsakteure mit nur indirektem Einfluss als Adressaten der Unternehmensverantwortung betrachten.

\subsubsection{Stakeholder als Adressaten der Unternehmensverantwortung}

Grundsätzlich werden zu den Adressaten der Verantwortung diejenigen Subjekte gezählt, denen gegenüber ein Unternehmen seine Verantwortung erklärt oder welche ein Unternehmen auf seine Verantwortung ansprechen (Fetzer 2004, S. 209). Potenziell sind dies all jene Personen oder Personengruppen, die sich von der Unternehmenstätigkeit in irgendeiner Art und Weise direkt oder indirekt betroffen fühlen und/oder Einfluss auf das Unternehmen haben bzw. auf deren Unterstützung das Unternehmen angewiesen ist (Rhenman 1968, S. 25). Diese werden auch als "Stakeholder" bezeichnet (Freeman 1984, S. 46).

Zu den möglichen Stakeholdern gehören demnach:

- Eigentümer ${ }^{10}$ (Anspruch auf Verzinsung des eingesetzten Kapitals, Mitspracherechte),

- Mitarbeiter und Führungskräfte (Anspruch auf Beschäftigung, Sicherheit, Gehaltszahlungen),

- Kunden/Klienten/Patienten (Anspruch auf Qualität, Zuverlässigkeit, Transparenz, Service),

- Lieferanten/Geschäftspartner (Anspruch auf Forderungsbegleichung und Stabilität der Geschäftsbeziehung) ${ }^{11}$,

- Kapitalmarkt, insbesondere Kreditgeber (Anspruch auf Zinsen und Tilgung),

- der Staat/die Behörden (Anspruch auf Steuergelder, Umweltschutz, Arbeitsplätze, Erfüllung eines ggf. bestehenden öffentlichen Auftrages) und

- die Öffentlichkeit (Parteien, Verbände, Kirchen, Medien, NGO's mit unterschiedlichen Ansprüchen).

Für das Krankenhaus sind zusätzlich zum Beispiel die Krankenkassen und niedergelassene Ärzte als

10 Sofern eine Trennung von Eigentum und Kontrolle vorliegt, das heißt angestellte Manager im Auftrag der Eigentümer die Geschäftsführung übernehmen.

11 Im Krankenhaus gehören dazu Pflegedienste, niedergelassene Ärzte, Pharmaunternehmen, Sanitätshäuser u.ä. 
wichtige Adressaten der Verantwortung zu nennen (s. Tab. 1 in Kap. 1).

Sowohl angestellte Führungskräfte als auch sonstige Mitarbeiter wurden in Kapitel 2.2.1 gleichzeitig als Verantwortungsträger klassifiziert, das heißt sie unterliegen einer Doppelrolle: Einerseits sind sie für die Wahrnehmung der Interessen anderer Stakeholder zuständig, andererseits haben sie eigene Ansprüche an das Unternehmen mit Blick auf gerechte Entlohnung und ein angemessenes Arbeitsumfeld (Göbel 2006, S. 121).

Nicht alle der genannten Gruppen werden tatsächlich von Unternehmen in gleicher Weise als Verantwortungsadressaten in Betracht gezogen. Vielmehr werden Stakeholder vielfach nach unterschiedlichen Kriterien klassifiziert und bewertet. Bei einer strategisch motivierten Bewertung geht es im Wesentlichen um das Einflusspotenzial und die Bedeutung verschiedener Stakeholdergruppen für den Unternehmenserfolg (Freeman 1984). Aus ethischer Sicht wird argumentiert, dass nur jene Personen/Personengruppen als Stakeholder verstanden werden sollten, die bereit sind, ihre Ansprüche argumentativ zu begründen und deren Anliegen als ,legitim“ erachtet werden (Ulrich 2001, S. 442; Fetzer 2004, S. 225). Legitim sind dabei in der Regel rechtlich begründete Interessen (z.B. auch alle auf vertraglichen Bindungen beruhenden Verpflichtungen) (Homann 1999, S. 54). Als wesentlich wird aber ein ethisches Bezugssystem für die Bewertung der Stakeholderanliegen betrachtet (Göbel 2006, S. 130). Ausgeschlossen werden zum Beispiel sozial disqualifizierte Anliegen wie zum Beispiel Lust an Sadismus, Zerstörung oder Herrschsucht (Höffe 2003, S. 21).

Welche Stakeholder für ein Unternehmen tatsächlich relevant sind, und welche Anliegen aus strategischer oder ethischer Sicht in Betracht gezogen werden, kann nicht allgemeingültig formuliert werden, sondern muss im Rahmen einer Stakeholderanalyse unternehmensspezifisch definiert werden (vgl. Kap. 3.3.2). Grundsätzlich soll im Folgenden der Ansicht gefolgt werden, dass die ethische Bewertung der Stakeholder seine Basis im Wertegerüst des Unternehmens findet (Freeman 1999, S. 234), wobei auf allgemein akzeptierte Bezugssysteme rekurriert werden sollte (z.B. Menschenrechte, Nachhaltigkeitsprinzip) (Göbel 2006, S. 131ff.). Auf dieser Grundlage verfolgt ein (strategisches) Stakeholdermanagement das Ziel, die unterschiedlichen Interessen potenziell relevanter Stakeholder in Einklang zu bringen, wobei ethische Kriterien für die Bewertung ihrer Anliegen mit berücksichtigt werden müssen.

\subsection{Dimensionen und Reichweite der sozialen Verantwortung}

\subsection{1 Überblick über Dimensionierungsansätze}

Die Reichweite des Konzeptes der sozialen Verantwortung kann über verschiedene Aspekte definiert werden:

- Erstens geht es darum, was von Unternehmen rechtlich und/oder aus sittlichen Vorstellungen heraus erwartet werden kann und muss und welcher Grad der normativen Verbindlichkeit durch das Verantwortungskonzept abgedeckt wird (Bindungscharakter der Normen bzw. Erwartungen).

- Zweitens geht es um die Frage, auf welche Themenbereiche sich das Konzept der sozialen Verantwortung erstreckt, das heißt den Grad der Verantwortung für ökonomische, soziale und/oder umweltrelevante Themen.

- Drittens geht es um den Bezug zur eigentlichen Unternehmenstätigkeit (dem Kerngeschäft) und die Übernahme von Verantwortung innerhalb oder außerhalb der Wertschöpfungskette des Unternehmens (begrenzt sich Verantwortung auf solche Aktivitäten, welche mit dem Kerngeschäft des Unternehmens unmittelbar verbunden sind, oder unterstützt das Unternehmen als Corporate Citizen mit entstandenen Gewinnen gesellschaftlich relevante Themen?).

- Und viertens geht es schließlich darum, inwieweit Unternehmen und die im Unternehmen handelnden Individuen nur für die unmittelbaren Konsequenzen ihrer Entscheidungen und Handlungen, auch für das institutionelle Gerüst, innerhalb dessen sie ihre Entscheidungen treffen, Verantwortung tragen.

\subsubsection{Dimensionierung über normative Bindungskraft}

Für eine Dimensionierung nach der normativen Bindungskraft der Erwartungen liefert das Konzept von Carroll eine gute Grundlage. Carroll (1979 und 1999) unterscheidet - neben der ökonomischen Verantwortung, die er als Grundbedingung 


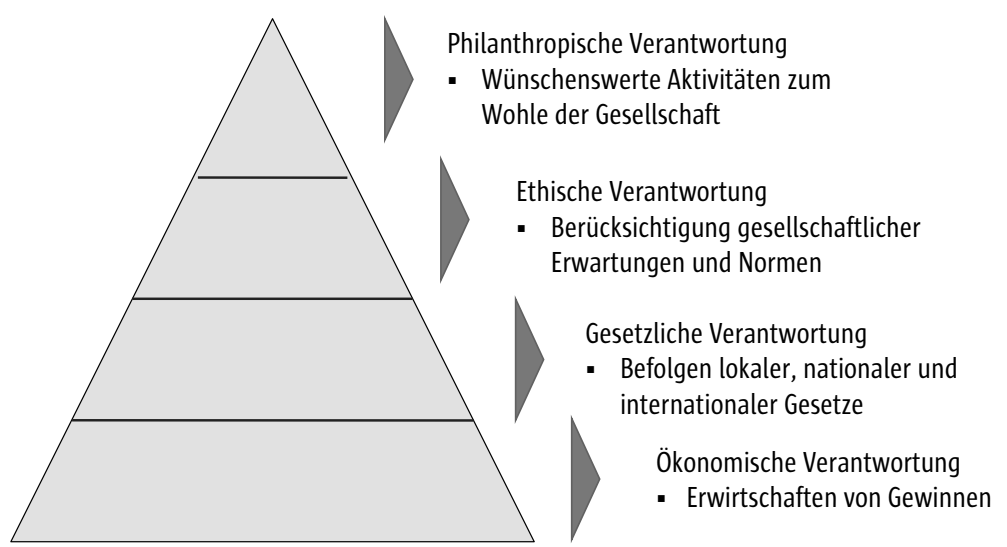

Abb. 6 Bereiche der Verantwortung (nach Carroll 1999)

für die Existenz von Unternehmen voraussetzt die rechtliche, die ethische und die philanthropische Verantwortung. Dabei ist die Gesetzestreue Pflicht; die ethische Verantwortung wird erwartet und die philanthropische Verantwortung gewünscht (vgl. Abb. 6).

Mit Bezug auf Kapitel 2.1.1, in welchem Freiwilligkeit als wesentliches Charakteristikum sozialer Verantwortung beschrieben wurde, würde die Sicherstellung rechtlich bindender Regeln (heute als „Compliance“ bezeichnet) vorausgesetzt werden und nicht unter das Konzept der sozialen Verantwortung fassen. Dies steht auch im Einklang mit der Ansicht, das sozial verantwortliches Handeln Handlungsspielräume voraussetzt, innerhalb derer die Handelnden unter Berücksichtigung ethischer Überlegungen und der Interessen der betroffenen Stakeholder Entscheidungen fällen (Schreyögg; Koch 2007, S. 59). Relevant für ein Management der sozialen Verantwortung sind aber in jedem Fall die ökonomische Verantwortung (als Voraussetzung und begründet durch die Erwartung der Eigentümer, Financiers und Mitarbeiter als Stakeholder) sowie die ethische Verantwortung. Bei philanthropischen Aktivitäten handelt es sich in der Regel um Aktivitäten der Gewinnverwendung (Spenden für soziale Zwecke oder die Umwelt, Corporate Volunteering Programme), welche zusätzlich zum Kerngeschäft durchgeführt werden. Im Unterschied zu der ethischen Verantwortung existiert dabei in der Regel kein Konflikt zwischen Stakeholdergruppen, der unter Anwendung ethischer Maßstäbe und im Dialog mit den Stakeholdern gelöst werden muss, sodass dieser Bereich häufig nicht der Unterneh- mensethik zugerechnet wird (Schreyögg 2007, S. 58). Nichtsdestotrotz nimmt das Unternehmen aber auch durch Mäzenatentum und Spenden eine gesellschaftliche Verantwortung ein und positioniert sich als „Corporate Citizen“, so dass auch dieser Bereich grundsätzlich zum Management sozialer Verantwortung gezählt wird.

In Zusammenhang mit dem Charakteristikum der Freiwilligkeit bzw. der Existenz von Handlungsspielräumen sind zwei Besonderheiten zu beachten. Einerseits ist aufgrund unterschiedlicher gesetzlicher Regelungen und ethischer $\mathrm{Er}-$ wartungen der Grad der normativen Bindung abhängig vom Ort der Unternehmensaktivität von einem Unternehmen wird in Europa anderes erwartet als in den USA oder in Asien. Andererseits ist die Existenz von Handlungsspielräumen abhängig von den beteiligten Akteuren im Unternehmen und kann von der Unternehmensführung gesteuert werden. Selbst wenn für das Unternehmen Handlungsspielräume bestehen, kann die Unternehmensführung durch interne Richtlinien ethisches Verhalten für die Mitarbeiter verbindlich machen oder über Leitlinien Hinweise geben, wie bei schwierigen Entscheidungen ethisch richtig verfahren werden könnte.

\subsubsection{Inhaltliche Dimensionierung}

Bei der inhaltlichen Abgrenzung geht es um Themenfelder, für welche Unternehmen eine Verantwortung tragen. Gemäß der in Kapitel. 2.1 genannten Definition bezieht sich das Konzept der 
sozialen Verantwortung auf soziale, ökologische und ökonomische Aspekt (s. Abb. 7).

Die ökonomische Verantwortung bezieht sich einerseits auf die Verpflichtung des Unternehmens gegenüber seinen Anteilseignern, Financiers und Mitarbeitern und gilt als Voraussetzung für weitere ökologische und soziale Aktivitäten. Andererseits fallen darunter auch freiwillige ökonomische Beiträge über das Kerngeschäft hinaus, wie zum Beispiel die Sicherung der Standortattraktivität. Die Verpflichtung zur Berücksichtigung ökologischer Aspekte kann aus den Präferenzen heutiger und zukünftiger Stakeholder abgeleitet werden. Eine Steuerung ökologischer Verantwortung vollzieht sich dabei über eine in-

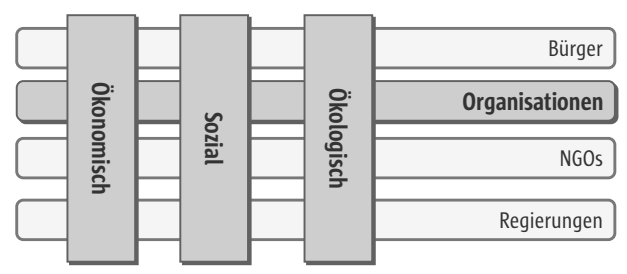

Abb. 7 Das 3-Säulen Konzept der unternehmerischen Verantwortung tensive Analyse der gesamten Wertschöpfungskette mit Blick auf die direkten und indirekten ökologischen Konsequenzen der Geschäftstätigkeit. Soziale Verantwortung schließlich bezieht sich auf die Verantwortung gegenüber sonstigen Personen bzw. Personengruppen und Institutionen, mit denen das Unternehmen direkt oder indirekt interagiert, das heißt Mitarbeiter, Kunden bzw. Patienten, Ärzte etc.

\subsubsection{Dimensionierung nach dem Bezug zur Unternehmenstätigkeit}

Die Dimensionierung nach dem Bezug zur Unternehmenstätigkeit bezieht sich im Wesentlichen darauf, wie weit die Verantwortung von Unternehmen über das eigentliche Kerngeschäft hinaus reicht bzw. reichen sollte (Hiß 2006, S. 38ff.; s. Abb. 8$)^{12}$.

12 Hiß sieht allerdings einen dritten inneren Verantwortungsbereich „Markt und Gesetz", welchen sie auch als „unfreiwillige“ CSR bezeichnet.

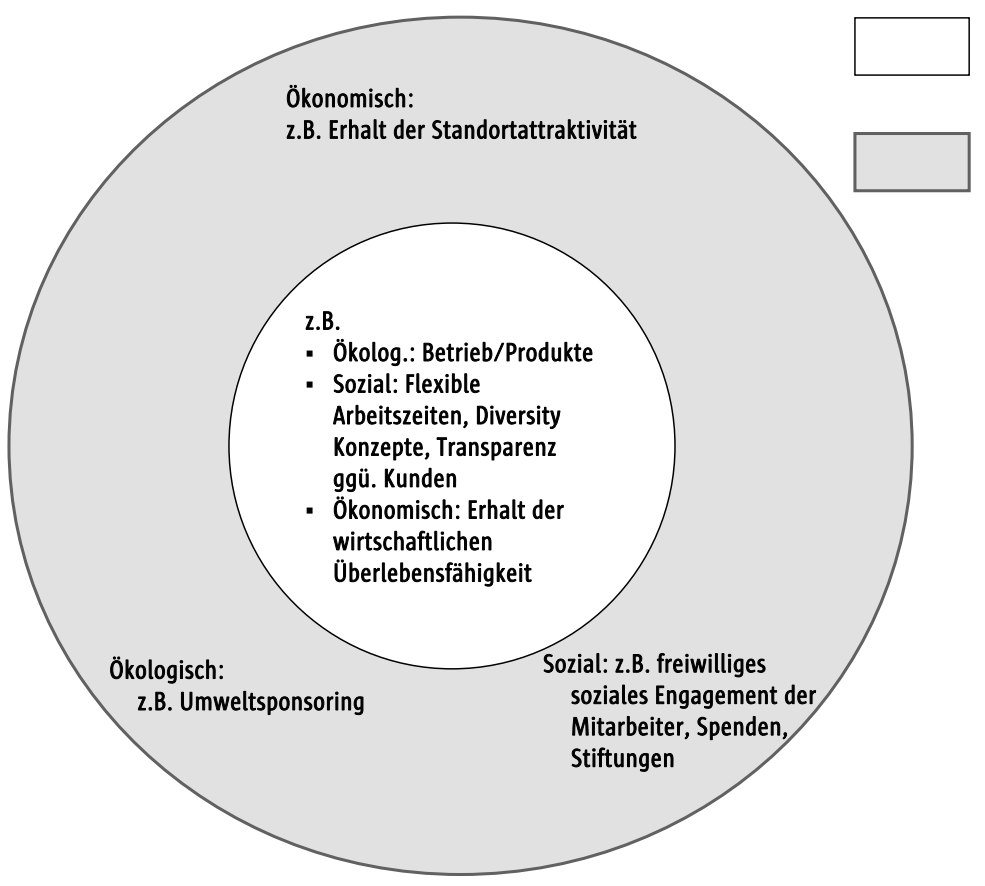

Unternehmerisches Kerngeschäft (Sustainable Business)

Unternehmerisches Umfeld (Corporate Citizenship)

Abb. 8 Verantwortung im Kerngeschäft und im unternehmerischen Umfeld 
Verantwortung innerhalb des Kerngeschäftes umfasst CSR-Aktivitäten, welche mit der Ausgestaltung der Wertschöpfungskette verbunden sind. Beispiele hierfür sind die Berücksichtigung ökologischer Aspekte im Produktionsprozess bzw. allgemeinem Betrieb (Verwendung umweltfreundlicher Materialien, Maßnahmen der Energieeinsparung, Reduktion von Emissionen u.ä.), Verhaltenskodizes oder soziale Gütesiegel. Im Kern muss dabei eine Unternehmenskultur stehen, welche den Verantwortungsgedanken unterstützt und lebt. Verantwortung außerhalb des Kerngeschäftes (auch als „äußerer Verantwortungsbereich" bezeichnet) umfasst philanthropische Aktivitäten, Mäzenentum, Spenden, Corporate Volunteering (Freistellung der Mitarbeiter für soziale Aktivitäten) und Public-Private Partnerships (kooperatives Zusammenwirken von Hoheitsträgern mit privaten Wirtschaftssubjekten). Dieser Verantwortungsbereich wird häufig auch als Corporate Citizenship bezeichnet (Schaltegger; Müller 2007, S. 22).

Initiativen der Wirtschaft (z.B. BAUM ${ }^{13}$ oder Econsense $^{14}$ ), welche sich mit Themen sozialer Verantwortung befassen, betreffen in der Regel beide Bereiche. Häufig geht es um einen Informationsaustausch bezüglich sinnvoller Maßnahmen und Vorgehensweisen oder aber um die Gestaltung von branchenweiten und branchenübergreifenden Standards, mit dem Ziel, dass sich verantwortliches Handeln zumindest nicht negativ auf die Wettbewerbsfähigkeit von Unternehmen auswirkt. Teilweise dienen die Initiativen dazu, engagierte Unternehmen mit sozialen Mittlerorganisationen zusammenzubringen, um so gemeinsame Projekte zu gestalten (z.B. UPJNetzwerk).
13 BAUM - Bundesdeutscher Arbeitskreis für umweltbewusstes Management, der 1984 als erste überparteiliche Umweltinitiative der Wirtschaft gegründet wurde.

14 Econsense - Forum Nachhaltige Entwicklung der Deutschen Wirtschaft e.V. ist ein Zusammenschluss führender global agierender Unternehmen und Organisationen der deutschen Wirtschaft zu den Themen Corporate Social Responsibility (CSR) und Nachhaltige Entwicklung (Sustainability). Das Unternehmensnetzwerk im Jahr 2000 auf Initiative des Bundesverbandes der Deutschen Industrie e.V. (BDI) gegründet - versteht sich als Think Tank und zentrale Dialogplattform.

\subsubsection{Ebenen der sozialen Verantwortung}

Unternehmerisches Handeln vollzieht sich auf drei Ebenen der Wirtschaft, welche miteinander in wechselseitigem Zusammenhang stehen (Enderle 1988, S. 55ff.):

- Die Mikroebene setzt sich zusammen aus den individuellen Wirtschaftsakteuren (insbesondere Konsumenten, Produzenten bzw. Dienstleistungsanbieter und Investoren), an deren Verhalten bestimmte Forderungen und Erwartungen geknüpft werden.

- Die Mesoebene konstituiert sich aus den Unternehmen als wirtschaftliche Organisationen. Das Unternehmen als Subjekt mit stabiler Identität kann Wirkungen an Dritten hervorrufen und beeinflusst über die innerbetriebliche Rahmenordnung die Entscheidungen der Unternehmensmitglieder. Gleichzeitig wirken Unternehmensmitglieder auch an der Gestaltung dieser Institutionen mit (Göbel 2006, S. 91ff.).

- Die Makroebene beschreibt die institutionellen Rahmenbedingungen des Wirtschaftens, das heißt Gesetze, Normen, Kultur, Institutionen etc. Die Rahmenordnung bestimmt den Handlungsspielraum von Unternehmen und weist ihnen bestimmte Rollen zu, wobei der Grad der Verbindlichkeit der Rollenerwartungen variiert (gesetzliche Ge- und Verbote versus kulturell geprägte gesellschaftliche Verpflichtungen). Gleichzeitig haben Unternehmen aber die Möglichkeit, die Spielregeln der Rahmenordnung zu beeinflussen, zu bestätigen oder zu verändern.

Insofern bezieht sich auch das Management der sozialen Verantwortung von Unternehmen auf alle drei Ebenen: Die Verantwortung für individuelle Entscheidungen und deren Folgen innerhalb eines gegebenen innerbetrieblichen oder überbetrieblichen institutionellen Rahmens, die Verantwortung für interne Strukturen, welche die Verantwortungsfähigkeit beeinflussen (Anreizsysteme, CSR Verantwortlichkeiten u.ä.) aber auch die Verantwortung für die außerbetrieblichen Spielregeln (z.B. Förderung der Entwicklung branchenweiter Standards), die verantwortliches Verhalten erleichtern oder erschweren können (Göbel 2006, S. 95ff., Homann, K./Gerecke, U. (1999), S. 453). 


\subsubsection{Grenzen der sozialen Verantwortung und Reichweite des Konzepts für das Krankenhausmanagement}

Das Konzept der sozialen Verantwortung verlangt, ethische Überlegungen in Unternehmensentscheidungen einfließen zu lassen. Es basiert auf dem Prinzip der Selbstverpflichtung und orientiert sich an den Erwartungen der Stakeholder, für welche Unternehmen sich verantwortlich erklären. Fraglich ist, wie wirkungsvoll das Konzept der Verantwortung tatsächlich im Unternehmensalltag sein kann und wo es seine Grenzen findet.

Unproblematisch ist die Übernahme von Verantwortung dann, wenn es gelingt, das unternehmerische Streben nach (langfristig) maximalen Gewinnen (Gutenberg 1961, S. 115) mit der Berücksichtigung der Interessen der Stakeholder vereinbar zu machen (sog. Win-win-Situationen) $)^{15}$. Freeman et al. (2007, S. 6) argumentiert in diesem Sinne und stellt die These auf, dass die Berücksichtigung der Interessen der Stakeholder gleichzeitig Ziel des Wirtschaftens und die Basis nachhaltigem ökonomischen Erfolges ist. Insbesondere in Fällen von Marktversagen (externe Effekte, öffentliche Güter, Informations- und Machtasymmetrien) kommt es jedoch häufig zu Konflikten zwischen unternehmerisch rationalem und kollektiv sinnvollem bzw. aus ethischer Sicht gebotenen Verhalten ${ }^{16}$. Teilweise können diese Konflikte gelöst werden, indem Unternehmen bewusst nach Möglichkeiten suchen, ökonomische und kollektive Rationalität vereinbar zu machen

- durch explizite Berücksichtigung zukünftiger Chancen, Risiken und Erträge, z.B. durch langfristigere Anreize innerhalb des Unternehmens (dies impliziert eine adäquate $\mathrm{Ge}$ staltung innerbetrieblicher Institutionen) oder

- durch Verlagerung des Problems auf eine andere Ebene, zum Beispiel die Forderung nach allgemein verbindlichen Standards zur Lösung des Free-Rider Problems oder nach Kontrollen

15 In diesem Fall würde das eigentliche Ziel des Wirtschaftens, nämlich das der bestmöglichen Versorgung von Gütern unter effizienter Ausnutzung der Ressourcen (materiales ökonomisches Prinzip) mittels individuell rationalem Verhaltens erreicht.

16 Ein gängiges Beispiel ist in diesem Fall das Prisoners Dilemma. und Offenbelegung zum Abbau von Informationsasymmetrien ${ }^{17}$.

Sind Konflikte zwischen ökonomischem Interesse und aus ethischer Sicht gebotenen Handlungen nicht lösbar, ist eine Abwägung zwischen ökonomischer Rationalität und ethischen Prinzipien erforderlich. Grundsätzlich kann gefordert werden, dass Unternehmen bzw. die handelnden Akteure sich auf Basis ihrer eigenen ethischen Maßstäbe ggf. zu Lasten von ökonomischem Erfolg moralisch verhalten. Allerdings sollten Verantwortungsträger bei der Wahrnehmung dieser Verantwortung ihre Entscheidungsfreiheit so nutzen können, dass sie durch die Folgen ihrer Entscheidungen nicht ihre langfristige Existenz gefährden (Homann/Suchanek 2005 S. 53ff.).

\section{Das Verständnis und die Reichweite der sozialen Verantwortung für das Krankenhausmanagement}

Im Rahmen des vorliegenden Beitrags liegt der Fokus auf den Kernpunkten des CSR Konzeptes, welche für den Bereich des Krankenhausmanagement Priorität haben. Erstens erfolgt ein Fokus auf den sozialen Aspekt; ökologische Herausforderungen und deren Berücksichtigung im Krankenhausmanagement werden zunächst nur am Rande behandelt. Außerdem sollte aus Sicht der Autoren der Bereich des Kerngeschäfts und die Beziehungen mit den wesentlichen Stakeholdern Priorität haben, philanthropische Aktivitäten bzw. Aktivitäten außerhalb des Kerngeschäfts werden zunächst außer Acht gelassen. Schließlich wird es im vorliegenden Beitrag weniger um „Compliance“ Aspekte, das heißt die Pflicht zur Befolgung gesetzlicher Regelungen, sondern vielmehr um die Berücksichtigung der Erwartungen der Stakeholder, sowie um den Umgang mit Erwartungskonflikten gehen.

Als wesentliche Verantwortungsträger werden im Folgenden das Unternehmen, sowie die im Unternehmen handelnden und entscheidenden Personen gesehen, deren Verantwortung sich sowohl auf individuelle Entscheidungen, als auch die Gestaltung innerbetrieblicher und die Beeinflussung überbetrieblicher Institutionen bezieht.

17 In diesem Sinne auch Albach (2003), der argumentiert, dass opportunistisches Verhalten in der Regel das Resultat ineffizienter Institutionen ist. 


\subsection{Begründung und Motive für ein Management sozialer Verantwortung}

\subsubsection{Ethische und strategische Begründungs- ansätze}

Unternehmen, Institutionen und auch Krankenhäuser sehen sich bei einer Vielzahl von Entscheidungen mit unterschiedlichen Erwartungen und Interessen verschiedenster Stakeholdergruppen konfrontiert. Es stellt sich die Frage, wie sie mit diesen Interessen umgehen, und wieweit sie welche Interessen berücksichtigen sollten.

Die Notwendigkeit eines Managements sozialer Verantwortung kann aus verschiedenen Überlegungen heraus abgleitet werden. Erstens lässt es sich begründen aus ethischen Überlegungen heraus bzw. einer „moralischen “ ${ }^{18}$ Pflicht zu verantwortungsvollem Handeln (Göbel 2006, S. 1820). Nicht nur Bürger, sondern auch andere gesellschaftliche Akteure haben Rechte und Pflichten und sind gehalten, sich „sittlich“ richtig bzw. gemäß gesellschaftlich anerkannten Normen und Werten zu verhalten. Verantwortungsvolles Management wird als Bedingung für die Berechtigung von Unternehmen und Institutionen gesehen (sogenannte „license-to-operate“). Aufgrund der unvollständigen Steuerungswirkung des Marktes ${ }^{19}$, des Rechtes und des Staates (s. Kap. 1.2) sind Ethik und Moral notwendig für ein funktionierendes Gesellschafts- und Wirtschaftssystems und führt verantwortungsvolles Verhalten in der Konsequenz langfristig zu besseren wirtschaftlichen Ergebnissen.

Zweitens kann die Notwendigkeit für verantwortliches Verhalten durch die Erwartung der Öffentlichkeit begründet werden. Gerade in den letzten Jahren und auch in Zusammenhang mit der derzeitigen Krise auf den Finanzmärkten wird immer mehr der Ruf nach Verantwortung, guter Unternehmensführung und moralischem Managerverhalten laut (Sahr 2009). Im Gesundheitswesen geht es einerseits um grundsätzliche Fragen der Medizinethik bzw. der klinischen Ethik (ethische Beurteilung von medizinischen Projekten, Behandlungsoptionen u.ä.) (Körtner 2007 S. 6ff.),

18 Moral wird dabei als System von Regeln, sittlichen Normen und Werten definiert, denen man als Mitglied einer sozialen Gemeinschaft unterliegt.

19 Als Resultat von Marktversagen aufgrund externer Effekte, öffentlicher Güter, unvollständiger Information und Machtasymmetrien andererseits aber um die Frage, wie existierende Ressourcenkonflikte in einer ethisch haltbaren Form gelöst werden können.

Drittens kann man schließlich die Verfolgung eines verantwortungsvollen Managementkonzeptes aus strategischen Überlegungen heraus begründen (Porter; Kramer 2006, S. 2). Verantwortungsvolles Verhalten von Unternehmen führt nicht nur zu einer Steigerung der Reputation gegenüber der Öffentlichkeit und den einzelnen Stakeholdern, sondern auch zu einer Stärkung der internen Erfolgspotenziale durch höhere Motivation der Mitarbeiter, Ressourcen sparendes Verhalten, größere Prozesseffizienz und höhere Innovationsfähigkeit von Unternehmen (Kim; Van Dam 2003, S. 9; Arthur D. Little 2003, S. 1). Die Verfolgung eines Managements sozialer Verantwortung kann also zu einer „Win-win-Situation" führen, von der alle Beteiligten profitieren (Margolis; Walsh 2001).

Unternehmen, welche ausschließlich als Antwort auf den Druck der Öffentlichkeit bzw. mit dem Ziel der Reputationsbildung verantwortlich handeln, laufen Gefahr, rein reaktiv zu handeln bzw. einen Fokus auf prestigeträchtige Marketingprojekte zu legen (Porter; Kramer 2006, S. 4). Ein Management sozialer Verantwortung, welches auf diesem Motiv beruht, kann daher nicht glaubwürdig sein.

Bei einem Vergleich der ethischen und der strategischen Begründung geht es im Wesentlichen um eine unterschiedliche Prioritätensetzung hinsichtlich der Bedeutung der ethischen Komponente bei Konflikten zwischen unterschiedlichen Stakeholder-Interessen bzw. zwischen moralisch richtigem und ökonomischem rationalem Handeln. Welchen Interessen stattgegeben wird, kann basierend auf ethischen oder wirtschaftlich-strategischen Überlegungen entschieden werden. Nach Homann/Blome-Dress ist es die Aufgabe von Unternehmen, ,möglichst weit in den Bereich mit hoher moralischer Akzeptanz vorzustoßen, ohne dabei die legitimen ökonomischen Zielsetzungen zu missachten“ (Homann; Blome-Drees 1992, S. 141), sodass im Wesentlichen nach lebensdienlichen und zugleich ökonomisch effizienten Lösungen gesucht werden muss (Göbel 2006, S. 147).

Unabhängig von ethischer oder strategischer Begründung beinhaltet das Management sozialer Verantwortung die Identifikation von Stakeholderanliegen, deren Priorisierung und die gezielte Suche nach einem Ausgleich zwischen unterschiedlichen Interessen (ebenda 2006, S. 65, 72). 


\subsubsection{Die Bedeutung eines Managements der sozialen Verantwortung für den Unternehmenserfolg}

Die meisten Verfechter des Konzepts der sozialen Verantwortung gehen von positiven ökonomischen Wirkungen verantwortungsvollen Handelns aus (z.B. Porter; Kramer 2006, Hansen; Schrader 2005, S. 384-386, Hansen 2004, S. 59ff.). Obschon die Zusammenhänge zwischen sozialer Verantwortung und Unternehmenserfolg meist nicht genau bekannt sind, werden positive Effekte durch eine höhere Reputation bei Stakeholdern einerseits sowie durch eine Stärkung interner Erfolgspotenziale angenommen (vgl. Abb. 9).

Zusätzlich wird der Einfluss auf das außerökonomische Umfeld über Kriterien wie Legalität, Legitimität und Handlungsspielraum genannt, welche wiederum indirekt den Unternehmenserfolg beeinflussen. (Dubielzig 2008, S. 216f.).

\section{Reputationswirkungen}

Zu den wichtigsten Stakeholdern zählen - neben der Öffentlichkeit - die Mitarbeiter, die Kunden

Stärkung der „internen“ Erfolgspotenziale

- Kostenreduktion (geringerer Ressourceneinsatz/effiziente Arbeitsabläufe)

- Erkennen langfristiger Chancen und Risiken/ Risikomanagement (Stakeholderdialog)

- Innovation (Stakeholder- und Wertkettenanalyse, Mitarbeitermotivation)

- Sicherung heutiger/zukünftiger Absatzmärkte (Zugang zu Netzwerken)

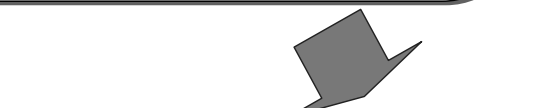

Positive Wirkungen auf Umsatz, Kosten, Rentabilität und Risiko bzw. Patienten und die wichtigsten Geschäftspartner des Unternehmens. Geschäftspartner des Krankenhauses sind insbesondere die niedergelassenen Ärzte, die Krankenkassen sowie die Behörden zu nennen. Verantwortliches Handeln führt dazu, dass ein Vertrauensverhältnis zu den Stakeholdern aufgebaut wird und Reputation geschaffen wird (Nijhof; Srnka 1999, S. 231f.). Dies wiederum kann dazu führen, dass erstens Kunden bzw. Patienten gewonnen und gebunden werden. Zweitens erleichtert das aufgebaute Vertrauen die Zusammenarbeit und die Verhandlungen mit Geschäftspartnern und Verbänden. Drittens sind potentielle Mitarbeiter leichter für das Unternehmen zu gewinnen und lassen sich aktuelle Mitarbeiter leichter halten. Und viertens schließlich sind Financiers eher bereit, in das Unternehmen zu investieren, entweder, da sie Vertrauen in die Nachhaltigkeit des Unternehmenserfolges haben oder da sie sich mit den Geschäftspraktiken identifizieren können.

\section{Stärkung interner Erfolgspotenziale}

Integriert man ökologische Aspekte in das Management sozialer Verantwortung, so lassen

Reputationsaufbau und -sicherung

- Öffentlichkeit („License to operate“)

- Kunden/Patienten (Differenzierung, Kundenbindung, Preissensibilität)

- Mitarbeiter (Recruiting, Commitment, Motivation)

- Sonstige Geschäftspartner (bessere Zusammenarbeit)

- Vereinigungen und Verbände (geringes Konfliktpotenzial bei Verhandlungen)

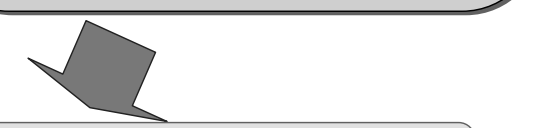

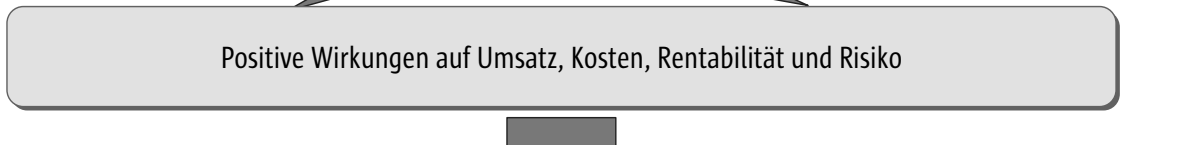

Größere Attraktivität für Investoren/Eigentümer und Fremdkapitalgeber

Abb. 9 Interne und externe Wirkungen des Verantwortungsmanagements 
sich häufig Kostenreduktionen durch geringeren Verbrauch von Energie, Wasser oder Materialien nachweisen (Schaltegger; Burritt 200o). Weiterhin führt der konsequente Dialog mit den Stakeholdern dazu, dass Risiken und Chancen frühzeitig erkannt und entsprechende Maßnahmen ergriffen werden können und Unternehmen eine größere Innovationskraft aufweisen. Wenn ein Unternehmen sich durch verantwortungsvolles Handeln auszeichnet, kann dies auch zu höherer Motivation der Mitarbeiter und damit zu besseren Leistungen führen; Mitarbeiter werden sich stärker mit dem Unternehmen identifizieren und sich engagieren (Nijhof; Srnka 1999, S. 232; Brands \& Values 2007, Studie Nr. 2). Schließlich wird darauf hingewiesen, dass CSR Maßnahmen - insbesondere wenn sie über ökonomische Beiträge das gesellschaftliche Umfeld unterstützen - heutige und neue Märkte sichern können (Peters 2009, S. 9).

\subsection{Die Umsetzung sozialer Verantwortung im Unternehmen}

\subsubsection{Ebenen der Berücksichtigung sozialer Verantwortung}

Wie in Kapitel 2.3.5 bereits diskutiert, muss das Management sozialer Verantwortung auf unterschiedlichen Ebenen angesiedelt werden.

Auf der Mikroebene geht es darum, innerhalb existierender Spielregeln sogenannte „Win-winSituationen " $z$ identifizieren bzw. zu gestalten, in welchen alle Beteiligten zumindest langfristig profitieren. Im Rahmen eines Strategieprozesses werden die Anliegen betroffener Stakeholder analysiert, Ziele gesetzt und entsprechende Strategien und Maßnahmen entwickelt, welche im besten Fall zu einer für alle akzeptablen Lösung führt (vgl. Ausführungen in Kap. 2.1.2).

Darüber hinaus sollten über die Verankerung der Werte in der Mission und den Leitlinien des Unternehmens, sowie die Anpassung von Strukturen, Prozessen und Systemen und die Etablierung einer entsprechenden Unternehmenskultur, die innerbetrieblichen Rahmenbedingungen so gestaltet werden, dass verantwortliches Handeln erleichtert bzw. unterstützt wird (Mesoebene) $)^{20}$.

20 Vgl. hierzu auch die Bausteine des um Nachhaltigkeitsaspekte erweiterten EFQM Modells für Sustainable Excellence (Sustainable
Schließlich kann Einfluss genommen werden auf die Spielregeln des Wettbewerbs bzw. die institutionellen Rahmenbedingungen (Makroebene), zum Beispiel durch aktive Forderungen nach gesetzlichen Regeln, welche verhindern, dass verantwortungsvolles Verhalten einzelner zu Wettbewerbsnachteilen führt (Strategie des „Ethical Displacements“ bzw. des Verlegens des Niveaus, DeGeorge 1993), oder durch Zusammenarbeit mit anderen Wettbewerbern zur Festlegung gemeinsamer Standards und Regelungen (Nijhof; Srnka 1999, S. 233).

\subsubsection{Die Elemente des Managements sozialer Verantwortung im Unternehmen}

Ziel eines Managements sozialer Verantwortung ist es, verantwortliches Handeln ganzheitlich im Unternehmen zu verankern und sich dabei konsequent an den Anliegen und Interessen der relevanten Stakeholder auszurichten. (s. Kap. 2.1.3) Die Idee der Verantwortung sollte Eingang finden in strategischen Zielen und Handlungspläne; ihre operative Umsetzung muss unterstützt werden durch entsprechende Strukturen, Systeme (Göbel 2006, S. 108f.) und personelle Ressourcen $^{21}$. Das Management sozialer Verantwortung im Unternehmen betrifft also im Wesentlichen drei Bereiche, welche im Rahmen eines integrativen Konzepts der Unternehmensführung miteinander verzahnt werden müssen (s. Abb. 10):

- Den strategischen und operativen Managementprozess, das heißt der analyse- und dialoggestützten Identifikation von Strategien und deren Umsetzung in strategischen Programmen und operativen Maßnahmen, sowie der Gestaltung von begleitenden Steuerungssystemen, die zu einem bestmöglichen Ausgleich unterschiedlicher Stakeholderanliegen führen.

Der strategische Managementprozess bildet die Basis der operativen Umsetzung und Planung; strategische Programme und interne Steuerungssysteme sind das Bindeglied zwischen strategischem und operativem Managementprozess. Im Folgenden erfolgt eine Konzentration auf den strategischen Prozess und

Excellence Group/Deutsche Bundesstiftung Umwelt 2006, S. 7-8) 21 Angelehnt an die Bereiche der Strategieimplementierung nach Schreyögg/Koch 2007, S. 115 


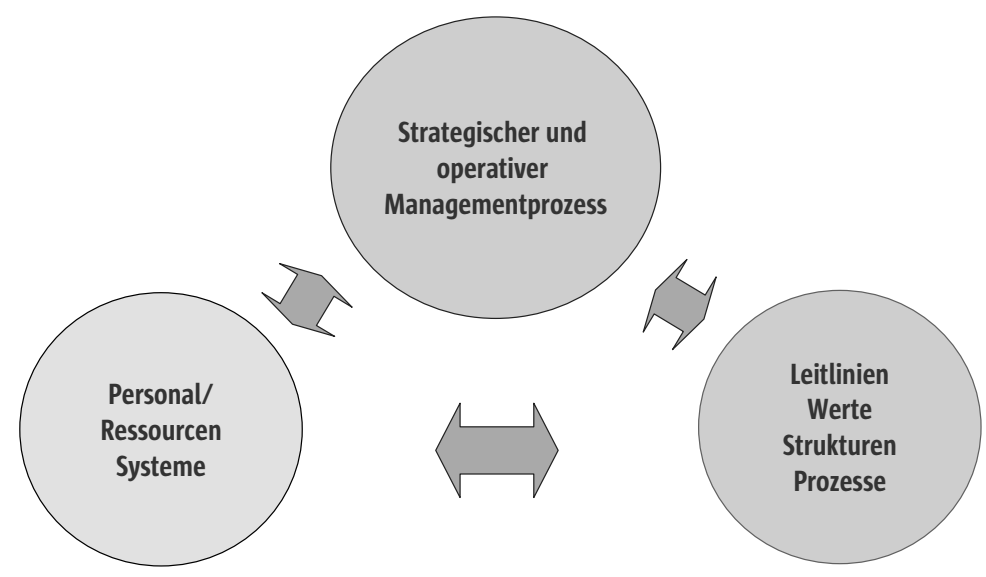

Abb. 10 Elemente des Managements sozialer Verantwortung

seinen Schnittstellen zum operativen Management (s. Kap. 3), ohne auf die operative Umsetzung weiter einzugehen.

- Die institutionelle Verankerung des Prinzips der Verantwortung in Strukturen (z.B. Ethik oder CSR Komitees oder Stabsstellen), Prozessen (z.B. Etablierung von Vorgehensweisen bei ethisch relevanten Entscheidungen), Systemen (Integration von relevanten Kennzahlen in unternehmenseigenen Systemen) sowie dem Leitbild eines Unternehmens, welches den Zweck des Unternehmens und die Verhaltensgrundsätze gegenüber Anspruchsgruppen definiert und so eine gemeinsame unternehmensweite Wertebasis schafft (s. hierzu Kap. 6 und 7).

Die Berücksichtigung der Grundsätze verantwortungsvoller Unternehmensführung im
Unternehmensleitbild begründet die Selbstverpflichtung des Unternehmens zu grundsatzkonformem Verhalten und bildet den Ausgangspunkt für den strategischen und operativen Managementprozess (Münstermann 2007, S. 38); er wird daher im folgenden Kapitel 3 mit behandelt (s. ferner dazu Kap. 7).

- Die Ressourcen und Kompetenzen, insbesondere die qualitative Personalstruktur: Anpassung der Anforderungen an Mitarbeiter und Führungskräfte mit Konsequenzen für die Mitarbeiterauswahl, -entwicklung und -führung. Wesentlich sind in diesem Zusammenhang auch die innerbetriebliche Kommunikation sowie die Gestaltung der Unternehmenskultur (s. Kap. 6). 